\title{
What is the real fair study model to compare open and laparoscopic mesh repairs for unilateral inguinal hernia regarding postoperative pain and discomfort?
}

\section{Opinion}

Inguinal hernia is a common surgical entity that consumes a large part of health resources worldwide. The discussion about the ideal surgical treatment is continued. Some authors strongly support endoscopic/laparoscopic repair (ELR) techniques. ${ }^{1}$ On the hand, open mesh techniques are considered to be easier to learn and performed with high success rates. ${ }^{2}$ Today, ELR's are generally named as 'minimally invasive'. However, general anesthesia is the rule for these procedures, while open mesh repairs can be done using local anesthesia (LA) in $90 \%$ of the cases. ${ }^{3}$ Total length of skin incisions for laparoscopic ports is also quite similar to that of an open procedure which is feasible via a $4-\mathrm{cm}$ incision. The classical Veteran Administration Multicenter Study comparing open and ELR's was published in 2004. ${ }^{4}$ The open technique was found to be superior to the laparoscopic technique for mesh repair of primary hernias. However, a Cochrane review and newer studies say that ELR's have an equal efficacy with open mesh repairs. ${ }^{5}$ ELR's have also been recommended for bilateral and recurrent inguinal hernias in the European Hernia Society (EHS) Guidelines in $2009,{ }^{6}$ and even for primary unilateral hernias in the recent update.?

The most important outcome measure following an inguinal hernia repair is recurrence. It has been reported that both ELR's and open repairs provide low recurrence rates. ${ }^{8}$ When it comes to postoperative pain, ELR's have generally been announced to provide less postoperative pain and quicker recovery in comparative clinical studies, ${ }^{9}$ but it is not easy to say that the promotion of ELR's by some surgeons, companies and even media including the internet does not create a bias in favor of these newer methods. Generally, surgeons tell their patients that ELR's are less invasive and related with less pain and quicker recovery. In a very recent questionnaire study about the patient perception of laparoscopic versus open mesh repairs, it was revealed that many patients had incorrect perceptions, a large number were under the false impression that laparoscopic techniques were safer, quicker and less likely to recurrence over open repair. ${ }^{10}$ Besides, many patients felt that laparoscopic repair was the only method which could be undertaken as a day case procedure. In fact, LA which is very suitable for open repair provides earlier discharge in comparison with general and even regional anesthesia that is required for ELR's. LA results, in comparative studies, in a higher degree of patient satisfaction than other anesthetic techniques. It also facilitates faster mobilization and earlier discharge/fulfillment of discharge criteria from post anesthetic care units than other anesthetic techniques. ${ }^{11,12}$ On the other hand, in a very recent prospective non-randomized study, Symeonidis and colleagues reported that transabdominal preperitoneal repair under spinal anesthesia was superior to open repair performed under different types of anesthesia in terms of immediate postoperative (24h) pain. ${ }^{13}$ However, repairs with spinal anesthesia resulted in a higher rate of urinary retention which could be eliminated by LA.

\author{
Volume 4 Issue 2 - 2017
}

\author{
Hakan Kulacoglu \\ Ankara Hernia Center, MD, FACS, Turkey
}

Correspondence: Hakan Kulacoglu, Cukurambar mahallesi, Budapeste Caddesi, 33/+Cankaya,Ankara,Turkey Fax +90 312 22000 90, Email hakankulacoglu@hotmail.com

Received: March 02, 2017 | Published: March 03, 2017

Another interesting finding in Patel et al's study is that most patients arrive at an outpatient appointment without a preferred method and possibly directed to laparoscopic repair, reflecting surgical practice of the institution. ${ }^{10}$ Most surgeons have their own strong views and preferences over one repair technique, and can convince their patients in favor of an approach. Pain and quicker recovery are subjective outcomes measures for scientific studies. Both are largely related to patients' feelings. More objective outcome measures in some controlled studies revealed that ELR's offer no advantage over open approach in respect of inflammatory and metabolic responses to surgery. ${ }^{14}$ However, most patients naturally think the newer technique is also the better. A newer and fancier technique naturally may provide a placebo effect for the patient regarding subjective complaints like pain and discomfort. This affect can be enhanced when his or her surgeon tells positively about a selected repair technique. If the patients with primary inguinal hernias use the search engines in the internet to get information about surgical treatment they will meet lots of pages promoting ELR's, including industry web sites. All these factors may easily create a bias in favor of ELR's. However, open mesh repair with LA provide very good results in our practice, and when a patient is told about the same advantages of an open mesh repair with LA he or she may experience very low pain after surgery. In addition, local infiltration anesthesia has a preemptive effect, diminishes the metabolic answer and lowers the pain in early postoperative period..$^{15}$ It is one of the main advantages of open repairs over ELR's. For this reason, LA is recommended for open repairs for not only frail patient who cannot tolerate general anesthesia but for every suitable case, as mentioned the update in EHS Guidelines in 2014. ${ }^{7}$ Bright et al reported that the frequency of attendance to chronic pain clinic after inguinal hernia repairs was significantly higher after laparoscopic repairs versus open repairs. ${ }^{16}$ However, $75 \%$ of the patient underwent an open repair in this retrospective study. Interestingly, chronic pain was more frequent after TEP which was least common repair (3\%) in that institution versus TAPP (performed in $22 \%$ ). A similar bias existed in the classical VA multicenter study. ${ }^{4}$ Laparoscopic repair had resulted in higher complication rates because of some centers where laparoscopic repair experience lacked. This may be the case again when the condition is reverse and worse outcomes can be reported by surgeons who are not familiar to Lichtenstein repair with LA. 
Currently, many surgeons and centers are seems to be subspecialized regarding the repair techniques for inguinal hernias. ${ }^{17,18}$ Some of them perform laparoscopic repairs routinely, whereas some others use only open repairs. Besides, most surgeons who prefer Lichtenstein repair use general anesthesia for this procedure, and do not offer the patients the advantages of LA. Nevertheless, one of the most important advantages of Lichtenstein repair is LA, and it should be used to obtain best results. However, a successful application of LA with intravenous light or mild sedation is the key for promising results. Pain is a feeling to learn and remember easily, if a patient feels pain during the surgery or in the immediate postoperative period because of inadequate LA he/she may develop acute and chronic pain following repair. Actually, many surgeons, especially senior ones are rather diffident about their capability of success in LA. ${ }^{19}$ We can compare this situation with that some older surgeons stick with conventional surgical techniques over newer ones like laparoscopic procedures. A good LA application for Lichtenstein repair requires formal courses like laparoscopy skill programs. LA education during residency is probably an important way to enhance the use of LA. Some European centers now have a residency program on LA for inguinal hernia repair. ${ }^{20}$ Residents could achieve comparable results with supervisor surgeons after a specific education, and senior surgeons may also be of benefit from similar educations. The Cochrane review by McCormack et al has shown that recovery is quicker with less persisting pain and numbness after laparoscopic repairs. ${ }^{5}$ However, the comparative studies for laparoscopic and open inguinal hernia repairs analyzed in this review are heterogeneous regarding anesthesia types. The number of studies with open mesh repair with LA is very limited. General and spinal anesthesia was used in many studies comparing laparoscopic and open mesh repairs. This means the lack of preemptive analgesic effect of LA for a Lichtenstein repair. There is only two specific studies in the PubMed comparing TEP under general anesthesia and Lichtenstein with local anesthesia. ${ }^{21,22}$ Dahlstrand and colleagues reported that unilateral inguinal hernia patients who underwent the laparoscopic TEP procedure suffered less pain 6weeks after inguinal hernia repair than those who underwent Lichtenstein repair with local anesthesia. ${ }^{21}$ Dhankhar et al also found that the operating time was significantly longer in the TEP group, whereas postoperative pain scores in the TEP group were lower than the scores in Lichtenstein group, but the difference was not statistically significant. As a surgeon who has specific interest in hernia repairs, generally do prefer Lichtenstein repair for unilateral inguinal hernias, follow the patients diligently at postoperative 1, 3, 6 and 12months, accept the concept of tailored approach in inguinal hernia repair, ${ }^{23}$ and is fully aware of the evidences in favor of ELR's in the systematic analyses and the guidelines, I can say that the rate of acute and chronic pain and discomfort postoperatively are very low after a Lichtenstein repair with LA. I consider LA as not only a good option for the repair procedure but also an advantageous one for less postoperative acute and chronic pain. I think, today, we should possibly be focused on an important question: What is the ideal study model to compare laparoscopic and open hernia repairs? Which study protocol provides a real fair comparison? It may be a model in which each technique is performed by its expert surgeons in its own optimal conditions. We can propose a prospective randomized controlled clinical study models to compare laparoscopic and open repairs for a primary unilateral inguinal hernia without bias. In this protocol, the patients in the one arm of the study undergo endoscopic/laparoscopic repair under general anesthesia, whereas the patients in the other arm are subjected to open mesh repair (Lichtenstein operation) using LA. Patients will be enrolled into two arms by using a computerized system. They are informed about the fact that both open and laparoscopic mesh repairs are safe and effective methods for inguinal hernias with low recurrence rates, no expression is made in favor of one specific technique. The patients who do not accept randomization and prefer the other technique will be excluded. Two surgeons who are experts in one of two procedures will perform all the repairs during the study period. Both surgeons will tell their patients the advantages of the operation he will perform. The outcome measures are postoperative pain and recovery time. An independent monitor is included in the study; he/she may be a surgeon or an anesthesiologist with a special interest in agrology. The monitor should see the patients blindly, with no information about the operative technique used. Follow up must last at least for one year.In my opinion, the most suitable and fair study that can compare ELR's and open repairs for primary inguinal hernias in respect of postoperative pain without a possible placebo effect and bias may be the presented model here. The results of this study after one year would renew and enhance our knowledge about the open and laparoscopic repair for inguinal hernias.

\section{Acknowledgements}

None.

\section{Conflict of interest}

The author declares no conflict of interest.

\section{References}

1. Balakrishnan S, Singhal T, Samdani T, et al. Laparoscopic inguinal hernia repair: over a thousand convincing reasons to go on. Hernia.2008;12:493-498.

2. Reuben B, Neumayer L. Surgical management of inguinal hernia. Adv Surg. 2006;40:299-317.

3. Kulacoglu H, Ozyaylali I, Yazicioglu D. Factors determining the doses of local anesthetic agents in unilateral inguinal hernia repair. Hernia. 2009;13:511-516.

4. Neumayer L, Giobbie-Hurder A, Jonasson O, et al. Veterans Affairs cooperative studies program 456 Investigators. Open mesh versus laparoscopic mesh repair of inguinal hernia. NEngl JMed. 2004;350:18191827.

5. McCormack K, Scott NW, Go PM, et al. Laparoscopic techniques versus open techniques for inguinal hernia repair. EU Hernia Trialists Collaboration Cochrane Database Syst Rev. 2003;1:CD001785.

6. Simons MP, Aufenacker T, Bay-Nielsen M, et al. European Hernia Society guidelines on the treatment of inguinal hernia in adult patients. Hernia. 2009;13(4):343-403.

7. Miserez M, Peeters E, Aufenacker T, et al. Update with level 1 studies of the European Hernia Society guidelines on the treatment of inguinal hernia in adult patients. Hernia. 2014;18(2):151-163.

8. Eklund AS, Montgomery AK, Rasmussen IC, et al. Low recurrence rate after laparoscopic (TEP) and open (Lichtenstein) inguinal hernia repair: a randomized, multicenter trial with 5-year follow-up. Ann Surg. 2009;249(1):33-38

9. Eklund A, Rudberg C, Smedberg S, et al. Short-term results of a randomized clinical trial comparing Lichtenstein open repair with totally extraperitoneal laparoscopic inguinal hernia repair. $\mathrm{Br} J \mathrm{Surg}$. 2006;93(9):1060-1068. 
10. Patel M, Garcea G, Fairhurst K, et al. Patient perception of laparoscopic versus open mesh repair of inguinal hernia, the hard sell. Hernia. 2012;16(4):411-415.

11. Maddern GJ, Rudkin G, Bessell JR, et al. A comparison of laparoscopic and open hernia repair as a day surgical procedure. Surg Endosc. 1994;8(12):1404-1408.

12. Callesen T. Inguinal hernia repair: anaesthesia, pain and convalescence. Dan Med Bull. 2003;50(3):203-218.

13. Symeonidis D, Baloyiannis I, Koukoulis G, et al. Prospective nonrandomized comparison of open versus laparoscopic transabdominal preperitoneal (TAPP) inguinal hernia repair under different anesthetic methods. Surg Today. 2014;44(5):906-913.

14. Schrenk P, Bettelheim $P$, Woisetschläger R, et al. Metabolic responses after laparoscopic or open hernia repair. Surg Endosc. 1996;10(6):628-632.

15. Paredes Esteban RM, Velasco Sánchez B, Martínez M, et al. Effect of the LA in the response to the pain. Study in the inguinal herniotomy. Cir Pediatr. 2008;21:162-166.

16. Bright E, Reddy VM, Wallace D, et al. The incidence and success of treatment for severe chronic groin pain after open, transabdominal preperitoneal, and totally extraperitoneal hernia repair. World J Surg. 2010;34(4):692-696.
17. Köckerling F, Berger D, Johannes OJ. What is a certified hernia center? The example of the German Hernia Society and German Society of General and Visceral Surgery. Front Surg. 2014;1:26.

18. Kulacoglu H, Oztuna D. Current status of hernia centres around the globe. Indian J Surg. 2014;77(s3):1023-1026.

19. Seker G, Kulacoglu $\mathrm{H}$. The acceptance rate of local anaesthesia for elective inguinal hernia repair among the surgeons working in a teaching hospital. J Coll Physicians Surg Pak. 2012;22(2):126-127.

20. Paajanen H, Varjo R. Ten-year audit of Lichtenstein hernioplasty under local anaesthesia performed by surgical residents. BMC Surg. 2010;10:24.

21. Dahlstrand U, Sandblom G, Ljungdahl M, et al. TEP under general anesthesia is superior to Lichtenstein under local anesthesia in terms of pain 6 weeks after surgery: results from a randomized clinical trial. Surg Endosc. 2013;27(10):3632-3638.

22. Dhankhar DS, Sharma N, Mishra T, et al. Totally extraperitoneal repair under general anesthesia versus Lichtenstein repair under local anesthesia for unilateral inguinal hernia: a prospective randomized controlled trial. Surg Endosc. 2014;28(3):996-1002.

23. Köckerling F, Schug-Pass C. Tailored approach in inguinal hernia repairdecision tree based on guidelines. Front Surg. 2014;1:20. 